\title{
Visualizing EBSD Maps With MTEX
}

\author{
Ralf Hielscher ${ }^{1}$, Florian Bachmann ${ }^{2}$ \\ 1. Department of Mathematics, Chemnitz University of Technology, Germany \\ 2. Department of Geoinformatics, Freiberg University of Technology, Germany
}

The visualization of measured data is a core challenge in the applied science. This is especially true in the context of EBSD data where spatially indexed orientations have to be visualized. In our talk we elaborate on the problem of assigning colors to orientations from a quite theoretical point of view but also demonstrate advanced visualization techniques for EBSD data using the open source texture analysis toolbox MTEX.

\section{The general problem}

The central problem is to find a mapping that associates to each orientation, i.e., to a class of crystallographically equivalent rotation matrices, a certain color such that the resulting EBSD map is easy to interpret. Ideally, such a mapping should satisfy the following requirements

1. similar orientations should have similar colors

2. different orientations should have different colors

3. important orientations, e.g. cube or brass, should have simple colors, e.g. red, blue, ...

The problem with requirement one is that the orientation space is three dimensional but the color space does not provide same contrast in three dimensions. Actually, it provides highest contrast only on the circle of saturated colors: red, purple, blue, green, yellow, and orange. The contrast in the two other dimensions, e.g., saturation and lightness is much less. The problem with requirement two is that the orientation space has no outer boundary, i.e., at any orientation there are neighbouring orientations in all three dimensions. This is not true for the color space where white, black and all saturated colors are boundary points.

In our presentation we will show that it is impossible to find a mapping that satisfies all three requirements. While the well known mapping that associates Euler angles to RGB values ignores requirement one the mappings that use colorizations of the inverse pole figure ignore requirement two. We believe that ignoring requirement 1 is more troublesome as, e.g., one grain might be interpreted as many grains just because the orientations of the grain are mapped to different colors. The jumps introduced by the Euler to RGB mapping are illustrated in Figure 1.

\section{Sharp textures}

The challenge when visualizing sharp textures, e.g. misorientations within one grain, is to constrict the contrast provided by the color space to a small subset of orientations. Surprisingly, this makes requirement two more simple to satisfy as the orientation space is locally Euclidean and its global structure does not matter in this context. As a consequence, a data dependent Euler angle to RGB mapping may satisfy all three conditions. However, for maximum contrast close to the modal orientation other mappings seems superior and will be discussed in the presentation. 


\section{Colorizing grain boundaries}

When analyzing the grain structure in EBSD maps one is often interested in visualizing the misorientations between neighbouring grains, e.g. in order to highlight twinning. Since a misorientation is again a class of crystallographically equivalent rotations the same reasoning as for plain orientation mapping applies. In our talk we explain how very specific colorizations of grain boundaries can be performed with the help of MTEX, e.g. according to

- misorientation angle / axis

- twinning / coincident site lattice

- tilt and twist boundaries

\section{Colorizing grains by other properties}

Finally we discuss how to colorize grains according to custom properties, e.g.

- shape properties

- kernel averaged misorientation (KAM)

- mechanic properties, like compressibility, active slip system

Along with these examples we demonstrate the capabilities of MTEX to create publication ready plots for a wide range of applications.

\section{References:}

[1] R. Hielscher, F. Bachmann, MTEX - A texture analysis software, www.mtex.googlecode.com
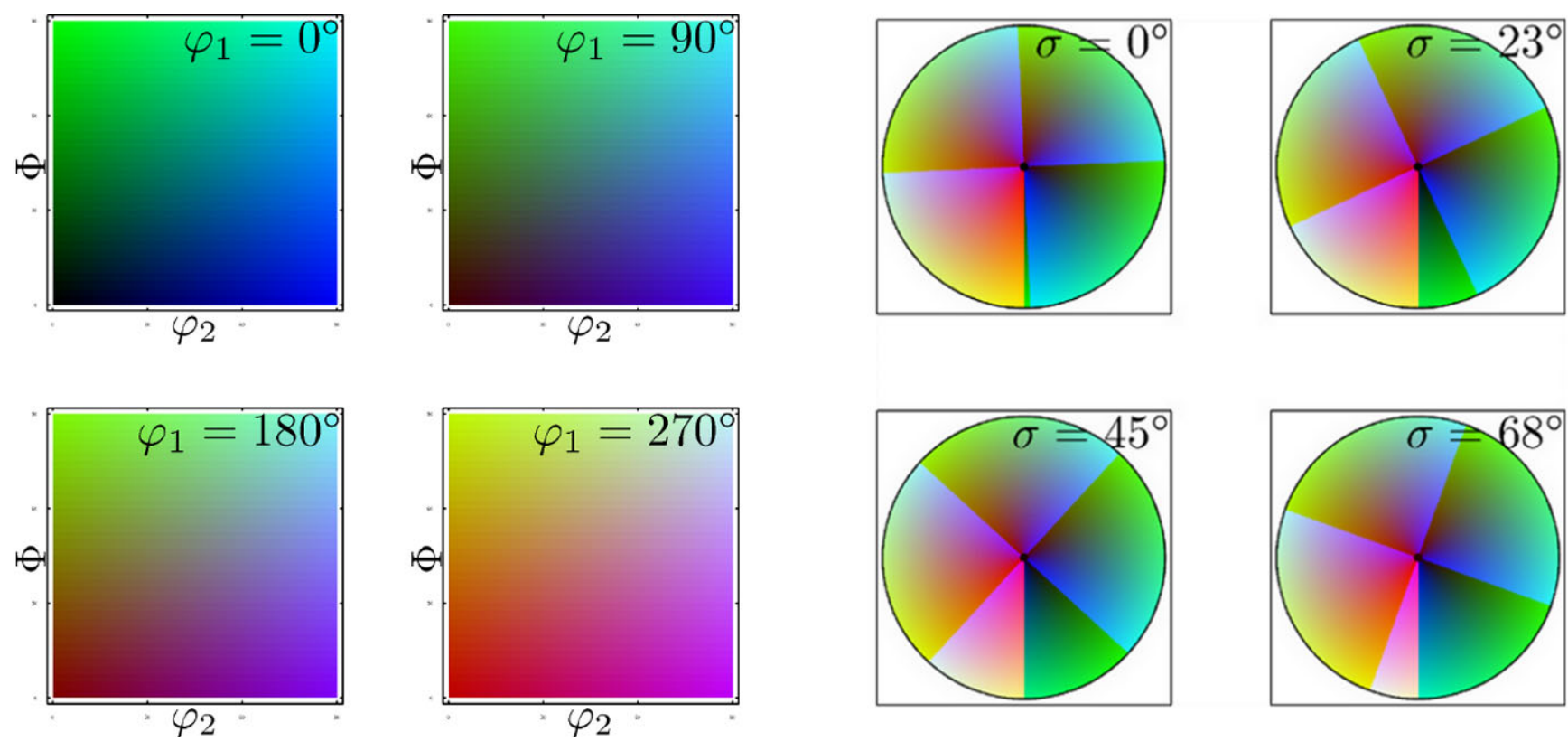

Figure 1. The orientation space colorized according to its Euler angles in phil and sigma sections. One clearly observes the discontinuities of the mapping in the sigma sections. 